\title{
Fast Fabrication of Colorful Nanostructures Using Imprinting with Femtosecond Laser Structured Molds
}

\author{
C.Y. Lin ${ }^{1}$, P.H. Wu ${ }^{1}$, K.P. Chang ${ }^{1}$, C.W. Cheng ${ }^{1 *}$, and S.M. Huang ${ }^{2}$ \\ ${ }^{1}$ ITRI South, Industrial Technology Research Institute, \\ No. 8, Gongyan Rd., Liujia District, Tainan 734, Taiwan R.O.C. \\ ${ }^{2}$ Central Mint, No.577, Jhensing Rd., Gueishan, Taoyuan 33353, Taiwan R.O.C. \\ E-mail: CWCheng@itri.org.tw (C.W. Cheng)
}

\begin{abstract}
Femtosecond laser induced periodic surface structures (FLIPSS) on many material surfaces have drawn much attention in recent years. However, the relatively low throughput of direct production process limits its potential of high volume manufacturing (HVM). In this study, fast replication of FLIPSS on Ag parts by imprinting process is demonstrated. An area of the high hardness (HRC 5860) cold work die steel SKD 11 is irradiated by femtosecond laser pulses with a top-hat beam profile to form periodic-like nanostructures with the periods of $600 \sim 700 \mathrm{~nm}$. It was then used for the process of imprinting. An Ag part with nano ripples can be replicated quickly, which proves that HVM of LIPSS on Ag parts is possible.

DOI:10.2961/jlmn.2012.01.0010
\end{abstract}

Keywords: femtosecond laser, LIPSS, nanostructures, imprinting

\section{Introduction}

Femtosecond laser machining has been recognized as an effective tool for materials surface micro/nano modification. It results less debris contamination, good reproducibility and minimal heat-affected zone. When irradiating a metal surface with femtosecond laser pulses, ripples or quasi-periodic nanostructures or self-organized structures can be formed in a simple one-step process $[1,2]$. This technique is referred to as femtosecond laser-induced periodic surface structure (FLIPSS). The formation of periodic nanostructures can be attributed to the interference between incident femtosecond laser wave and the surface scattered wave [3-6] and also the two plasmon decay models [7].

FLIPSS technique has many interesting applications such as fabrication of ultra-low reflectance metal surfaces [8-11], polarization-controlled stainless steel coloration [12], surface wettability tuning of silicon [13-15], glass [16], and metal [17-21]. However, the relatively low throughput of femtosecond laser processing limits the potential of high volume manufacturing. Mass production techniques, such as injection molding, hot embossing, and imprinting process are suitable for plastic or metallic parts.

In this study, fast replication of colorful nanostructures on Ag parts by imprinting using a SKD11 steel die has been demonstrated. The die was first irradiated by top-hat linearly polarized femtosecond laser pulses for nanostructure generation and then used for cold imprinting. An Ag part with surface nanostructures was replicated quickly, which proves that HVM of LIPSS on Ag parts is possible.

\section{Experimental}

The polished SKD 11 steel die was machined using a 120 fs pulse duration regenerative amplified Ti:Sapphire laser system (Spitfire, Spectra-Physics). The central wavelength is $800 \mathrm{~nm}$ and the repetition rate is $1 \mathrm{kHz}$. The linearly polarized laser beam power is continuously adjustable in terms of pulse energy by a automated rotatable half- wave plate and a polarizing beam splitter. The Gaussian laser beam profiled was transformed into a top-hat one by using a beam shaper (piShaper, MolTech). The top-hat beam profile is shown in Fig. 1. The laser beam is focused onto the steel die surface at normal incidence by an objective lens (Mitutoyo 0.26 NA, M Plan Apo NIR). The positioning of the die was done by using a three-axes precision stage. The fabrication process was monitored continuously via a coaxial machine vision system. The surface profile of the LIPSS processed die was observed by using a scanning electron microscopy (SEM).

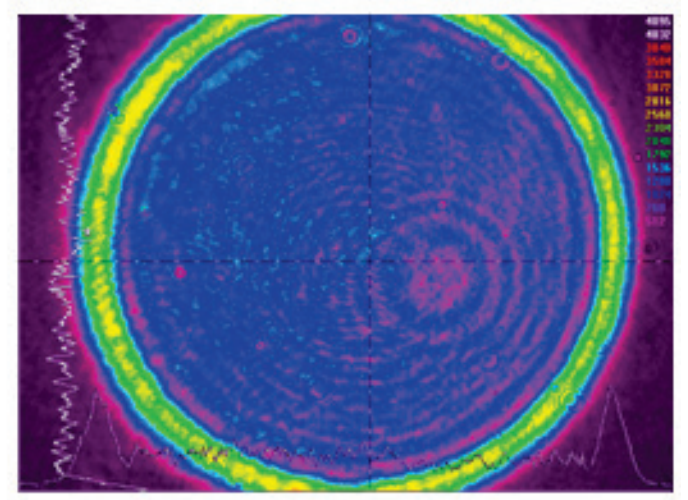

Fig. 1 The top-hat beam profile.

\section{Results and Discussion}

Fig. 2 presents a SEM image of a single point irradiated by 100 laser pulses at the fluence of $0.48 \mathrm{~J} / \mathrm{cm}^{2}$. The profile reflects the irradiation intensity of the top-hat distribution as was shown in Fig. 1. Fig. 3 corresponds to the same laser fluence with different number of laser shots from 10 to 100. It is observed that the appearance of the periodic-like nanostructures is dependent on the number of laser shots received per area. The periodic-like nanostructure evolves with the increasing number of pulses. Previous studies [22] 
have shown that the processing of diamond-like carbon film using stationary linearly polarized femtosecond laser pulses, the nanostructures also evolves with the increasing number of pulses

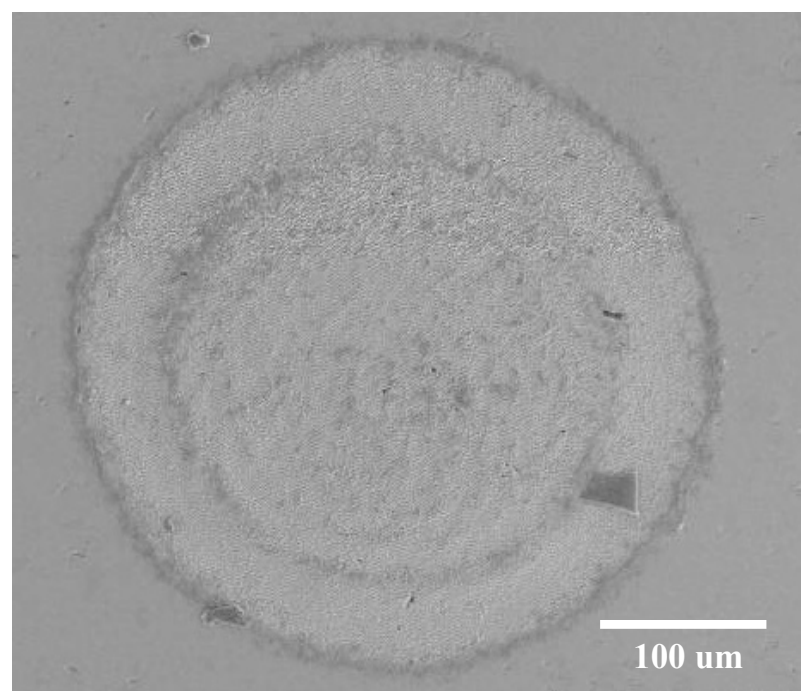

Fig. 2 SEM image of a single-point pattern irradiated at the laser fluence of $0.48 \mathrm{~J} / \mathrm{cm}^{2}$ with 100 shots.

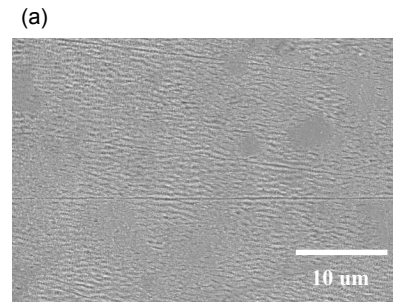

(c)

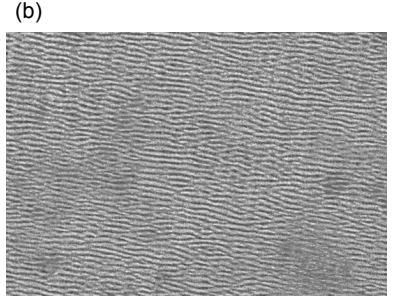

(d)

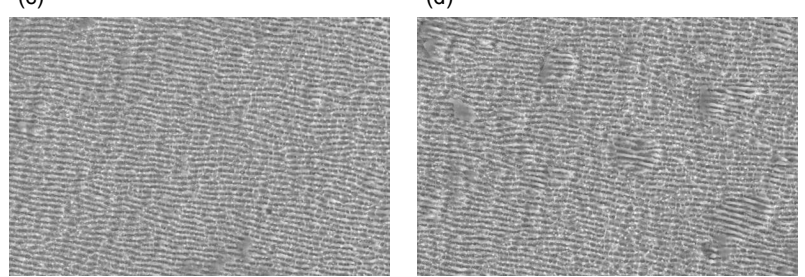

Fig. 3 SEM images of a central area of the single-point pattern irradiated at the laser fluence of $0.48 \mathrm{~J} / \mathrm{cm}^{2}$ with (a) 10 , (b) 20 , (c) 70 , and (d) 100 shots.

Fig. 4(a) presents a low magnification SEM image of the line pattern irradiated at the laser fluence of $0.48 \mathrm{~J} / \mathrm{cm}^{2}$ with a scanning speed of $760 \mathrm{~mm} / \mathrm{min}$. The scanning direction is perpendicular to the laser polarization. The magnified images of areas A and B are shown in Fig. 4(b) and (c), respectively. As seen from Fig. 4(b), nanostructures with a period of approximately $600 \sim 700 \mathrm{~nm}$ were formed, which was slightly less than the laser wavelength at $800 \mathrm{~nm}$. In addition, the orientations of the periodic nanostructures were nearly perpendicular to the polarization direction (E). The result agrees with previous studies when linearly polarized femtosecond laser beam was used [4, 5, 23]. In Fig. 4(d), it can be seen that a two-scale morphology consisting of micro- and nanostructures was fabricated [6]. The nanostructures similar to that in Fig. 4(b) are on top of the microstructure. Fig. 5 shows the SEM images of a line pattern written at the laser fluence of $0.48 \mathrm{~J} / \mathrm{cm}^{2}$ and scanning speeds of $800,780,740$, and $700 \mathrm{~mm} / \mathrm{min}$. It is observed that the appearance of the periodic nanostructures is inde-

pendent on the scanning speed, i.e. on the number of irradiation laser pulses received per area.
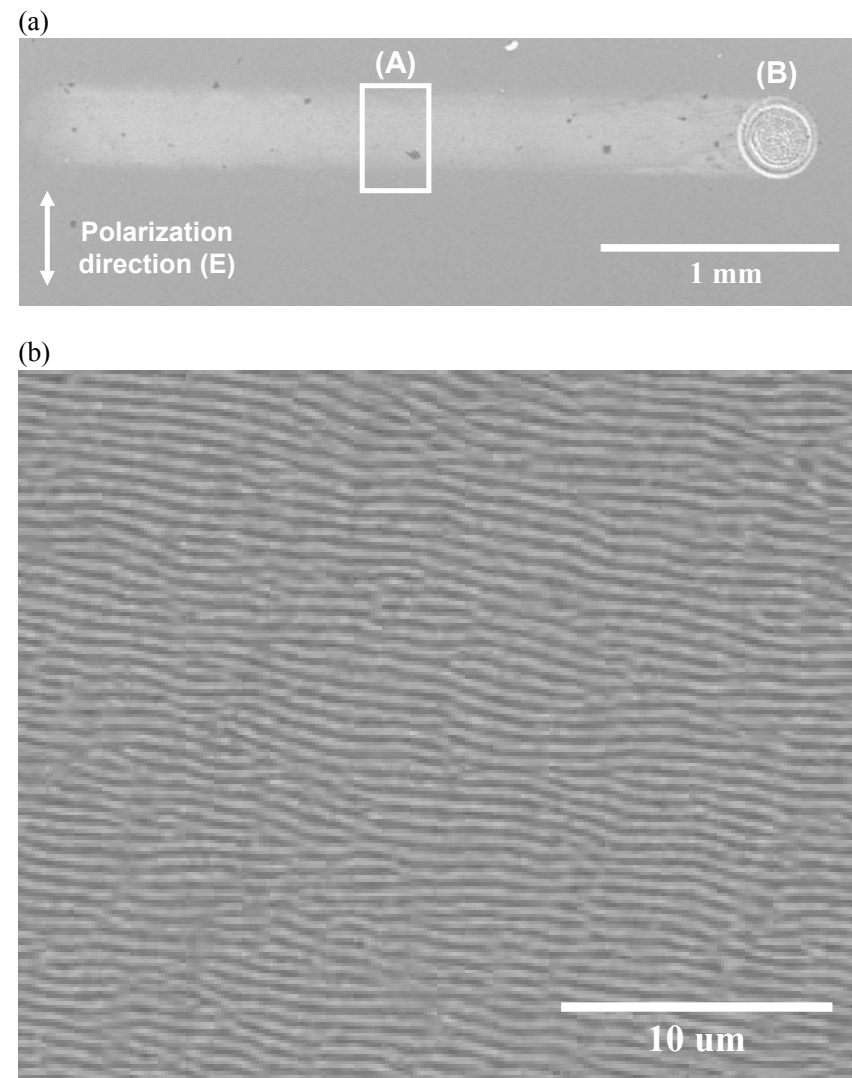

(c)

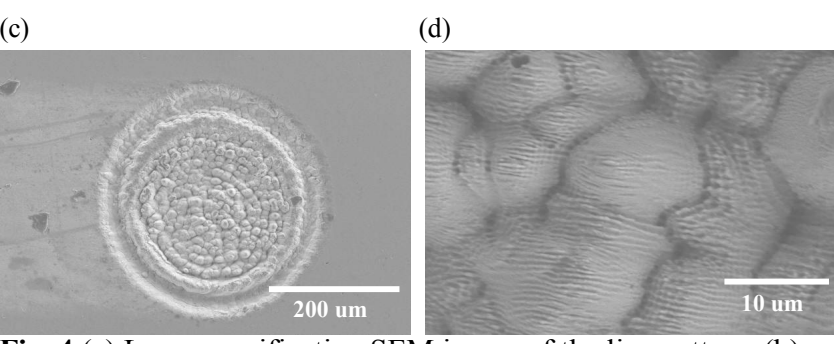

Fig. 4 (a) Low magnification SEM image of the line pattern, (b) magnified SEM image of the area A, (c) magnified SEM image of the area B, and (d) the central area in Fig. 4 (c).

(a)

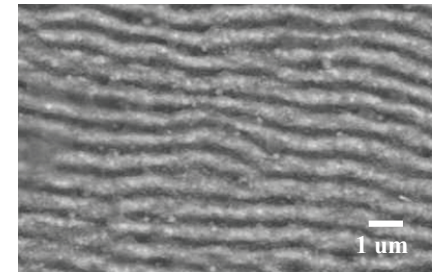

(c)

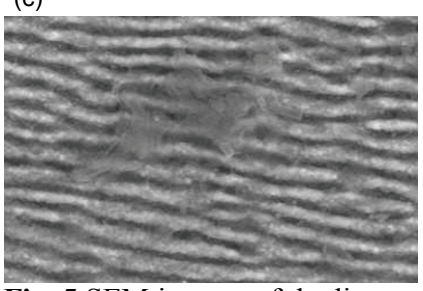

Fig. 5 SEM images of the line pattern written at the laser fluence of $0.48 \mathrm{~J} / \mathrm{cm}^{2}$ and scanning speeds of (a) 800 , (b) 780, (c) 740, and (d) $700 \mathrm{~mm} / \mathrm{min}$. (b)

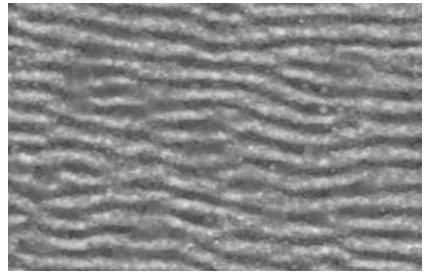

(d)

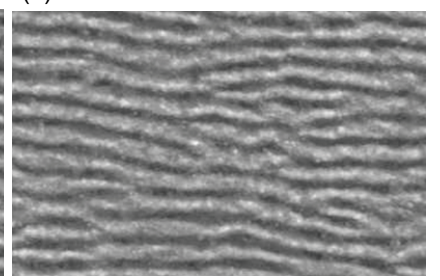


Fig. 6(a) shows a photograph of a $20 \times 20 \mathrm{~mm}^{2}$ area on the SKD 11 steel die irradiated at the fluence of $0.48 \mathrm{~J} / \mathrm{cm}^{2}$ and the scanning speed of $740 \mathrm{~mm} / \mathrm{min}$. Its line pattern is similar to that shown in Fig. 5(c). Fig. 6(b) shows the imprinted Ag part from the die (imprinting pressure 10.58 $\mathrm{N} / \mathrm{cm}^{2}$ ). The colorful reflection can be observed on the laser-irradiated area on both the die and imprinted part. This is due diffraction of illumination white light by the nanostructures. Fig. 6(c) presents SEM image from the square area in Fig. 6(b). The nanostructures were imprinted on the Ag part. The line pattern is not as clear as those shown in Fig. 4(b), which is believed because of the debris of the Ag material got stocked in the die during the imprinting process. However, from Fig. 6(b), the diffraction effect of light is still visible. The imprinted $\mathrm{Ag}$ part is metal colorized without its painting. Our finding proves that HVM of LIPSS color metal is viable.

(a)

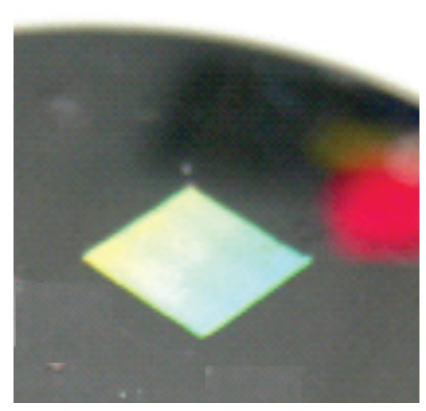

(b)

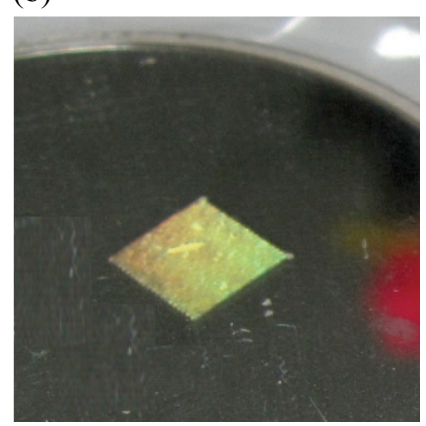

(c)

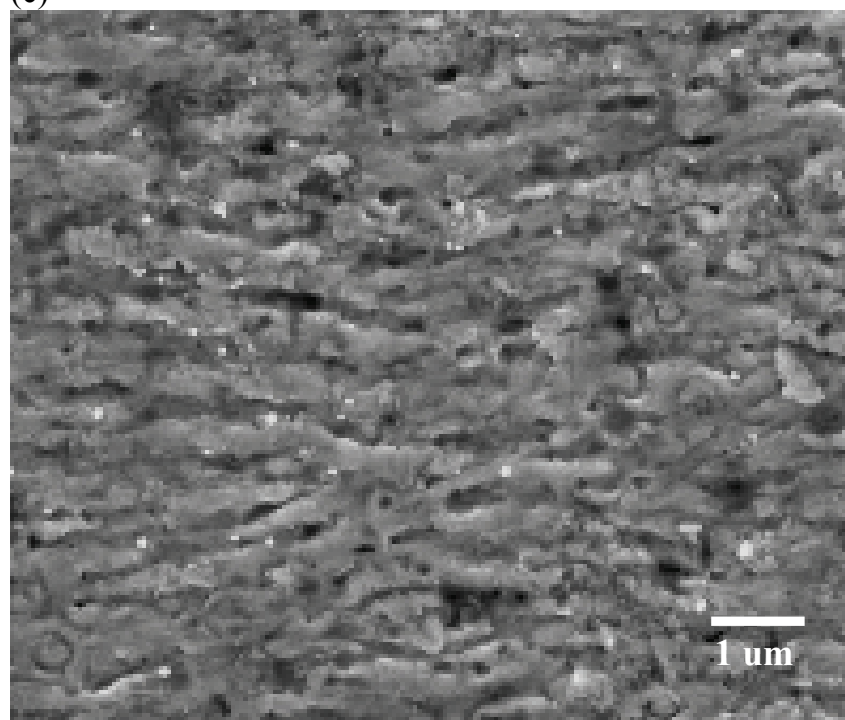

Fig. 6 Photograph of the femtosecond laser irradiated (a) SKD 11 steel die (b) imprinted Ag part, and (c) SEM image of the square area in Fig. 6 (b)

\section{Conclusions}

This paper has reported a novel low-cost and fast fabrication process for periodic nanostructures using imprinting with a femtosecond laser surface-textured metal die. The SKD 11 steel die was fabricated by the FLIPSS technique using a femtosecond laser with a top-hat beam profile. An array of nanostructures was formed on $\mathrm{Ag}$ parts by cold imprinting process. The desired color effect on the imprint- ed part proves that HVM of FLIPSS color metal without paint is possible.

\section{Acknowledgements}

We would like to appreciate the support from the Ministry of the Economic Affairs (MOEA), Taiwan, R.O.C. for performing this project.

\section{References}

[1] L. T. Qi, K. Nishii, and Y. Namba, "Regular subwavelength surface structures induced by femtosecond laser pulses on stainless steel," Optics Letters, vol. 34, no. 12, pp. 1846-1848, 2009.

[2] B. K. Nayak, and M. C. Gupta, "Self-organized micro/nano structures in metal surfaces by ultrafast laser irradiation," Optics and Lasers in Engineering, vol. 48, no. 10, pp. 940-949, Oct, 2010.

[3] M. Hashida, S. Namba, K. Okamuro et al., "Ion emission from a metal surface through a multiphoton process and optical field ionization," Physical Review $B$, vol. 81, no. 11, 2010.

[4] M. Huang, F. Zhao, Y. Cheng et al., "Origin of LaserInduced Near-Subwavelength Ripples: Interference between Surface Plasmons and Incident Laser," Acs Nano, vol. 3, no. 12, pp. 4062-4070, Dec, 2009.

[5] J. Bonse, A. Rosenfeld, and J. Krueger, "On the role of surface plasmon polaritons in the formation of laser-induced periodic surface structures upon irradiation of silicon by femtosecond-laser pulses," Journal of Applied Physics, vol. 106, no. 10, Nov 15, 2009.

[6] M. Huang, F. Zhao, Y. Cheng et al., "The morphological and optical characteristics of femtosecond laser-induced large-area micro/nanostructures on GaAs, $\mathrm{Si}$, and brass," Optics Express, vol. 18, no. 23, pp. A600-A619, Nov 8, 2010.

[7] S. Sakabe, M. Hashida, S. Tokita et al., "Mechanism for self-formation of periodic grating structures on a metal surface by a femtosecond laser pulse," Physical Review B, vol. 79, no. 3, 2009.

[8] A. Y. Vorobyev, and C. Guo, "Enhanced absorptance of gold following multipulse femtosecond laser ablation," Physical Review B, vol. 72, no. 19, 2005.

[9] Y. Yang, J. J. Yang, C. Y. Liang et al., "Ultrabroadband enhanced absorption of metal surfaces structured by femtosecond laser pulses," Optics Express, vol. 16, no. 15, pp. 11259-11265, 2008.

[10] B. K. Nayak, M. C. Gupta, and K. W. Kolasinski, "Formation of nano-textured conical microstructures in titanium metal surface by femtosecond laser irradiation," Applied Physics a-Materials Science \& Processing, vol. 90, no. 3, pp. 399-402, 2008.

[11] V. V. Iyengar, B. K. Nayak, and M. C. Gupta, "Ultralow reflectance metal surfaces by ultrafast laser texturing," Applied Optics, vol. 49, no. 31, pp. 59835988, 2010.

[12] B. Dusser, Z. Sagan, H. Soder et al., "Controlled nanostructrures formation by ultra fast laser pulses for color marking," Optics Express, vol. 18, no. 3, pp. 2913-2924, 2010. 
[13] T. Baldacchini, J. E. Carey, M. Zhou et al., "Superhydrophobic surfaces prepared by microstructuring of silicon using a femtosecond laser," Langmuir, vol. 22, no. 11, pp. 4917-4919, 2006.

[14] V. Zorba, L. Persano, D. Pisignano et al., "Making silicon hydrophobic: wettability control by twolengthscale simultaneous patterning with femtosecond laser irradiation," Nanotechnology, vol. 17, no. 13, pp. 3234-3238, 2006.

[15] V. Zorba, E. Stratakis, M. Barberoglou et al., "Tailoring the wetting response of silicon surfaces via fs laser structuring," Applied Physics a-Materials Science \& Processing, vol. 93, no. 4, pp. 819-825, 2008.

[16] M. Zhou, H. F. Yang, B. J. Li et al., "Forming mechanisms and wettability of double-scale structures fabricated by femtosecond laser," Applied Physics aMaterials Science \& Processing, vol. 94, no. 3, pp. 571-576, 2009.

[17] M. N. W. Groenendijk, and J. Meijer, "Surface microstructures obtained by femtosecond laser pulses," Cirp Annals-Manufacturing Technology, vol. 55, no. 1, pp. 183-186, 2006.

[18] B. Wu, M. Zhou, J. Li et al., "Superhydrophobic surfaces fabricated by microstructuring of stainless steel using a femtosecond laser," Applied Surface Science, vol. 256, no. 1, pp. 61-66, Oct, 2009.

[19] G. Romer, A. J. H. in't Veld, J. Meijer et al., "On the formation of laser induced self-organizing nanostructures," Cirp Annals-Manufacturing Technology, vol. 58, no. 1, pp. 201-204, 2009.

[20] A. M. Kietzig, S. G. Hatzikiriakos, and P. Englezos, "Patterned Superhydrophobic Metallic Surfaces," Langmuir, vol. 25, no. 8, pp. 4821-4827, 2009.

[21] A. M. Kietzig, S. G. Hatzikiriakos, and P. Englezos, "Ice friction: The effects of surface roughness, structure, and hydrophobicity," Journal of Applied Physics, vol. 106, no. 2, 2009.

[22] G. Miyaji, and K. Miyazaki, "Origin of periodicity in nanostructuring on thin film surfaces ablated with femtosecond laser pulses," Optics Express, vol. 16, no. 20, pp. 16265-16271, Sep 29, 2008.

[23] P. Bizi-Bandoki, S. Benayoun, S. Valette et al., "Modifications of roughness and wettability properties of metals induced by femtosecond laser treatment," Applied Surface Science, vol. 257, no. 12, pp. 52135218, 2011.

(Received: June 5, 2011, Accepted: December 29, 2011) 